\title{
Concomitant, sequential, and 7-day triple therapy in first-line treatment of Helicobacter pylori infection in Korea: study protocol for a randomized controlled trial
}

Hyuk Lee ${ }^{1 \dagger}$, Beom Jin Kim² ${ }^{2 \dagger}$, Sang Gyun Kim³ ${ }^{3}$ Jin $\| \mathrm{Kim}^{4}$, II Ju Choi ${ }^{5}$, Yong Chan Lee ${ }^{6}$, Jae G. Kim², Jae J. Kim ${ }^{1 *}$ and Korean College of Helicobacter and Upper Gastrointestinal Research

\begin{abstract}
Background: Most international guidelines recommend triple-therapy regimens consisting of a proton pump inhibitor, clarithromycin, and amoxicillin/metronidazole for at least 7 days for the eradication of Helicobacter pylori. However, the efficacy of 7-day clarithromycin-based standard triple therapy for $\mathrm{H}$. pylori infection is currently unacceptable in Korea. In this study, we will compare the efficacy and safety of 7-day standard triple therapy, 10-day sequential therapy, and 10-day concomitant therapy for the first-line treatment of H. pylori infection in Korea.

Methods/design: In this multicenter, investigator-blinded, randomized trial we are recruiting adult patients with $H$. pylori infection from 15 hospitals in Korea to determine whether sequential or concomitant treatment is superior to standard triple therapy. Patients are randomly assigned to receive either standard triple therapy (lansoprazole, amoxicillin, and clarithromycin) for 7 days, or sequential treatment (lansoprazole and amoxicillin for the first 5 days, followed by lansoprazole, clarithromycin, and metronidazole for another 5 days) for 10 days, or concomitant therapy (lansoprazole, amoxicillin, clarithromycin, and metronidazole) for 10 days. The primary outcome is the rate of H. pylori eradication in the intention-to-treat population.
\end{abstract}

Discussion: The results of this study will be crucial for determining the optimal regimen for the primary treatment of $H$. pylori infection in Korea. This study will produce vital evidence that will lead to revisions to guidelines concerning first-line treatment regimens for $H$. pylori infection.

Trial registration: Clinical Research Information Service (CRIS), Republic of Korea, KCT0001980. Registered on 25 July 2016. Keywords: Helicobacter pylori, Triple therapy, Concomitant therapy, Sequential therapy

\section{Background}

Helicobacter pylori infection causes peptic ulcers, gastric mucosa-associated lymphoid tissue lymphoma, and gastric cancer [1]. Most international guidelines recommended triple-therapy regimens consisting of a proton pump inhibitor (PPI), clarithromycin, and amoxicillin/

\footnotetext{
*Correspondence: jgkimd@cau.ac.kr; jjkim@skku.edu

${ }^{\dagger}$ Equal contributors

${ }^{2}$ Department of Internal Medicine, Chung-Ang University College of Medicine, 102 Heukseok-ro, Dongjak-gu, Seoul 06973, South Korea

${ }^{1}$ Department of Medicine, Samsung Medical Center, Sungkyunkwan

University School of Medicine, 81 Irwon-ro, Gangnam-gu, Seoul 06351, South Korea

Full list of author information is available at the end of the article
}

metronidazole for at least 7 days for the eradication of H. pylori [2-4]. However, the eradication frequency of standard triple therapy has fallen to less than $80 \%$ in many countries because of the rising prevalence of clarithromycin resistance [5-7]. The rate of clarithromycin resistance is reported to be as high as $37.3 \%$ in Korea. Consequently, the first-line eradication rate of triple therapy has been shown to be $74 \%$ according to a national database [8,9]. Despite this issue, the standard triple-therapy regimen is still recommended for first-line therapy in Korea according to the revised guidelines, and it is the only regimen reimbursed by the Korean National Health Insurance Service [10]. 
Several strategies, including bismuth-containing quadruple therapy and non-bismuth-containing quadruple therapy (either sequential or concomitant), have been proposed to increase the eradication rate in Korea [11-14]. Because the efficacy of sequential treatment, which consists of a PPI and amoxicillin for the first 5 days, followed by a PPI plus clarithromycin and metronidazole for another 5 days, appears to be less susceptible to clarithromycin resistance than triple therapy, it was expected to become the standard first-line treatment for $H$. pylori infection. A meta-analysis indicated that sequential therapy was more effective than 7-day triple therapy, but there are regional differences in the efficacy of this approach, owing to geographical variations in antibiotic resistance $[15,16]$. Furthermore, the pooled eradication rates of sequential therapy as a first-line treatment are reported to be suboptimal in Korea [17]. Nonbismuth quadruple therapy is another alternative to concomitant therapy, and it is proven to work even when clarithromycin resistance is present [18]. Several randomized trials comparing sequential with concomitant treatment have indicated no significant differences, whereas sequential therapy was found to be slightly more effective in Central and South America $[19,20]$. In a small number of randomized controlled studies in Korea, 10-day or 5-day concomitant therapy was shown to be more effective than standard triple therapy [21, 22]. Some retrospective studies have demonstrated that concomitant therapy is superior to standard triple therapy [23]. Concomitant and sequential therapies were demonstrated to be similarly effective in a few head-to-head comparison studies in Korea [13]. Despite these previous results, evidence supporting these conclusions is insufficient because no studies with an adequately rigorous design have been performed. With these considerations in mind, we designed the present nationwide, population-based, randomized controlled trial to compare the efficacy of 10-day concomitant therapy, 10-day sequential therapy, and 7-day triple therapy as first-line therapy for $H$. pylori infection in a Korean population.

\section{Methods/design}

\section{Trial design and study setting}

This study is designed as a multicenter, randomized, three-arm, superiority, parallel-group trial. Participants will be randomly allocated to the following three arms in a 1:1:1 ratio:

1. Arm 1 (control arm): The standard triple-therapy group will receive lansoprazole $30 \mathrm{mg}$, amoxicillin $1 \mathrm{~g}$, and clarithromycin $500 \mathrm{mg}$ twice daily for 7 days.

2. Arm 2 (experimental arm 1): The sequential therapy group will receive lansoprazole $30 \mathrm{mg}$ and amoxicillin $1 \mathrm{~g}$ twice daily for the first 5 days, followed by lansoprazole $30 \mathrm{mg}$, clarithromycin $500 \mathrm{mg}$, and metronidazole $500 \mathrm{mg}$ twice daily for the remaining 5 days.

3. Arm 3 (experimental arm 2): The concomitant therapy group will receive lansoprazole $30 \mathrm{mg}$, amoxicillin $1 \mathrm{~g}$, clarithromycin $500 \mathrm{mg}$, and metronidazole $500 \mathrm{mg}$ twice daily for 10 days.

Participants will be recruited from 15 hospitals in Korea (Samsung Medical Center, Chung-ang University Hospital, Yonsei University Severance Hospital, National Cancer Center, Bundang Seoul National University Hospital, Chungbuk National University Hospital, Chungnam National University Hospital, Chonnam National University Hospital, Chonbuk National University Hospital, Chilgok Kyungpook National University Hospital, Keimyung University Dongsan Hospital, Pusan National University Hospital, Kosin University Gospel Hospital, Jeju National University Hospital, and Hallym University Chuncheon Sacred Heart Hospital) (Fig. 1). The recruitment period began in November 2016 and will end in October 2018. We received approval to conduct this study from the institutional review boards of 15 nationwide hospitals. This investigator-initiated trial was approved by the Korean Food and Drug Administration and is registered at https://cris.nih.go.kr/cris (KCT0001980). A flow diagram of the trial is shown in Fig. 2.

\section{Study subjects}

Consecutive outpatients with $H$. pylori infection aged $\geq$ 19 years who have undergone endoscopic examination within the prior 3 months, agree to trial participation, and provide written informed consent fulfill the eligibility criteria and are invited to participate in the study. Exclusion criteria include a history of $H$. pylori eradication therapy; gastric surgery; abnormal liver function test or renal function test results on screening laboratory studies; antibiotic use within the prior 4 weeks; PPI use within the prior 2 weeks; histamine- 2 receptor antagonist, aspirin, nonsteroidal anti-inflammatory drug, steroid, anticholinergic, prostaglandin analog, promotility drug, or sucralfate use within the prior week; use of drugs such as lovastatin, simvastatin, atorvastatin, indinavir, ritonavir, cyclosporin, terfenadine, cisapride, pimozide, astemizole, HIV protease inhibitors, and ergot alkaloids; allergic reactions to the study medications; and serious concomitant illnesses. In addition, we will exclude pregnant participants or participants who plan to become pregnant as well as patients participating in other clinical studies or who are otherwise deemed unsuitable by the researchers. Withdrawal criteria include protocol violations such as detection of eligibility violations, use of any forbidden medication during the trial that could influence the study results, or occurrence 


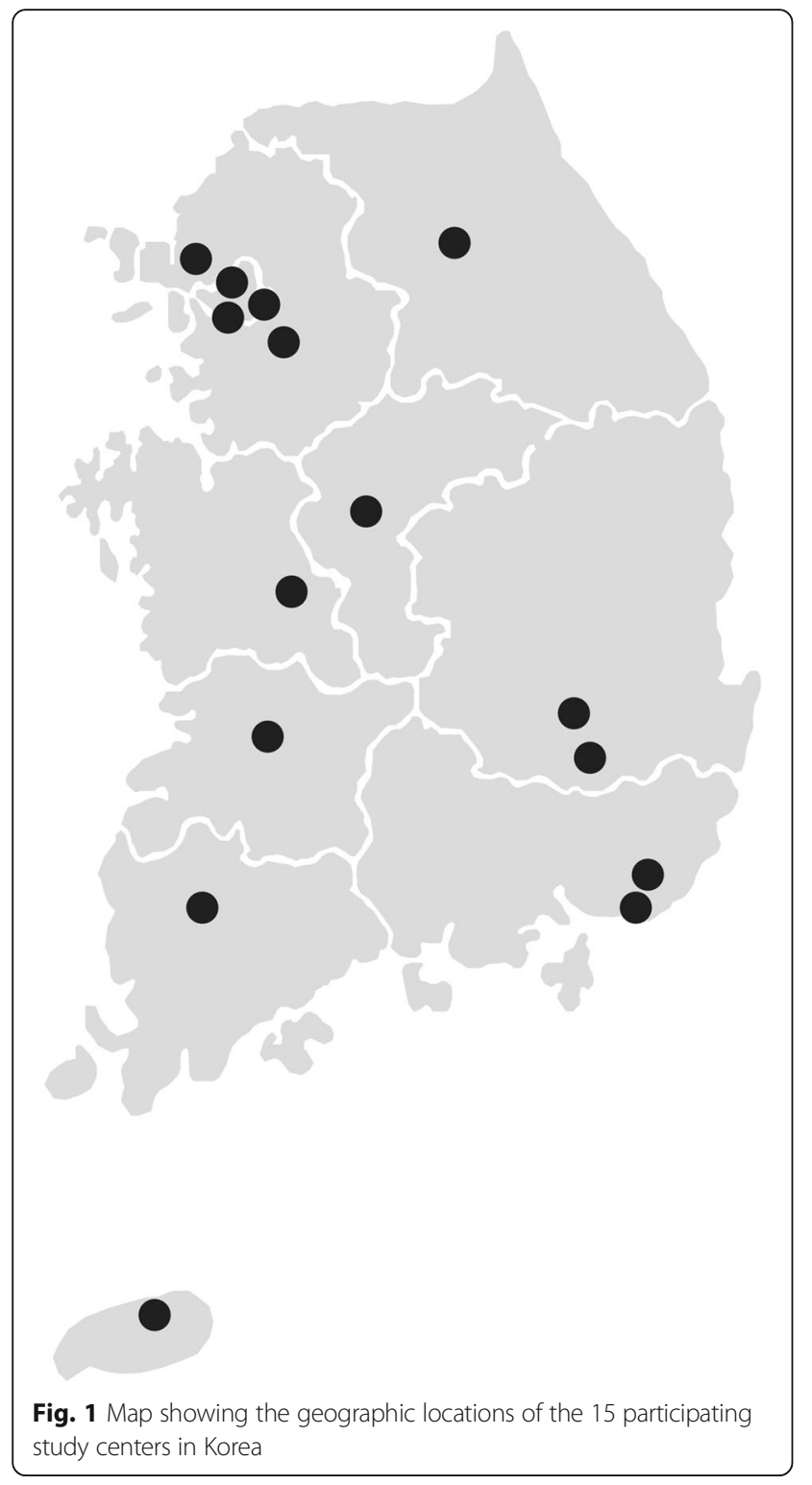

of other significant protocol violations. In addition, subjects with serious adverse events, an allergic reaction to the investigational product, withdrawal of consent, and decision to terminate for reasons of the subject's health will be withdrawn from the study. Symptomatic patients will be allowed to use antacids on demand in the posttreatment period. Antibiotics or other medications affecting the treatment results will be prohibited during the study period.

\section{Randomization and masking}

Randomization will be conducted using a centralized, web-based randomization system managed by the Medical Research Collaborating Center (https://mrcc.snu h.org) of Seoul National University Hospital, which uses permuted block randomization with a concealed and varying block size. Only the researcher at the Medical Research Collaborating Center is able to know which number indicates which treatment arm. To ensure concealed allocation, an independent staff member will dispense consecutively numbered, identically designed treatment packs that contain sealed bottles of study drugs. Physicians who are unaware of treatment assignment will manage all study participants. Participants will not be blinded to group allocation.

\section{Procedures}

The standard protocol for this study is shown in Fig. 3. Before enrollment, $H$. pylori infection status will be determined by a rapid urease test, urea breath test, histology, and/or bacterial culture. The patients will complete a standard questionnaire to collect complete demographic data, including age, sex, medical history, family history, history of smoking and alcohol consumption, and gastrointestinal symptoms. The patients will receive a printed handout that provides directions for taking the medications correctly and how best to adhere to treatment. The follow-up period of this study after randomization will be 8 weeks and will include two visits with each subject (first visit at 3 or 4 weeks after allocation, second visit 5-8 weeks after allocation). Adverse events and compliance will be evaluated at the first visit after allocation. At the second visit, the efficacy of $H$. pylori eradication therapy will be determined. In all subjects, a urea breath test will be conducted to assess $H$. pylori status at the fourth to sixth week after the end of $H$. pylori eradication therapy. A staff member blind to the eradication arm of each patient will perform the urea breath tests. The cutoff values will be set at $2 \%$ for the capsule urea breath test and $4.8 \%$ for the conventional urea breath test. Eradication will be defined as a negative result for the urea breath test. A Standard Protocol Items: Recommendations for Interventional Trials (SPIRIT) checklist is provided as an additional file (Additional file 1).

\section{Tolerability and adherence}

Drug tolerance and patient compliance will be evaluated at the end of treatment. Drug tolerance will be investigated by questioning patients about possible side effects, such as nausea, vomiting, dry mouth, diarrhea, constipation, rash, dizziness, disorientation, and candidiasis. Before treatment, the patients will be informed of the common adverse events associated with the study drugs and will be asked to record these symptoms during treatment in provided diaries. The adverse events will be assessed according to a 4-point scale: none, mild (discomfort annoying but not interfering with daily life), moderate (discomfort sufficient to interfere with daily life), and severe (discomfort resulting in discontinuation of eradication 


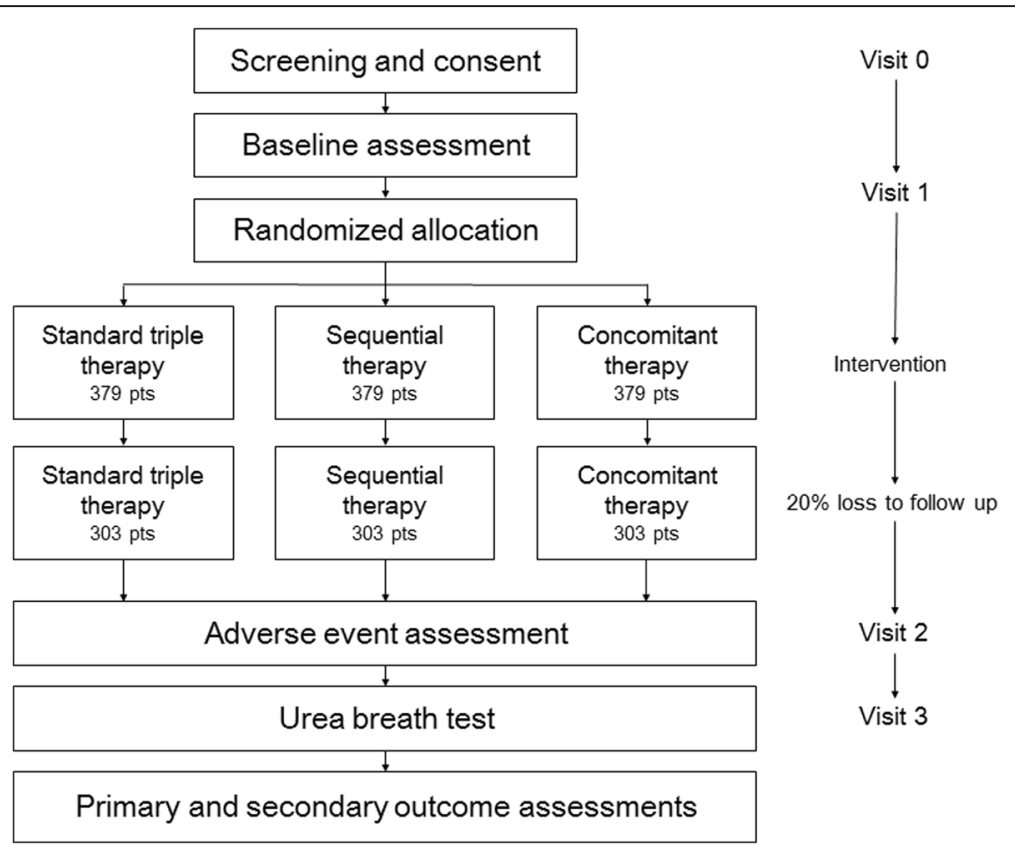

Fig. 2 Study design

\begin{tabular}{|c|c|c|c|c|c|c|c|c|c|c|}
\hline & Enrolment & Allocation & \multicolumn{8}{|c|}{ Post-allocation (weeks) } \\
\hline TIMEPOINT & $\begin{array}{c}\text { Before } \\
\text { randomization }\end{array}$ & 0 & 1 & 2 & 3 & 4 & 5 & 6 & 7 & 8 \\
\hline Enrollment & $x$ & & & & & & & & & \\
\hline $\begin{array}{r}\text { Eligibility } \\
\text { screen }\end{array}$ & $x$ & & & & & & & & & \\
\hline $\begin{array}{r}\text { Informed } \\
\text { consent }\end{array}$ & $x$ & & & & & & & & & \\
\hline Allocation & & $x$ & & & & & & & & \\
\hline Intervention & & & $x$ & $x$ & & & & & & \\
\hline Assessments & & & & & & & & & & \\
\hline $\begin{array}{l}\text { Baseline } \\
\text { variables }\end{array}$ & $x$ & & & & & & & & & \\
\hline $\begin{array}{l}\text { Primary } \\
\text { outcome }\end{array}$ & & & & & & & $x$ & $x$ & $x$ & $x$ \\
\hline $\begin{array}{r}\text { Secondary } \\
\text { outcome }\end{array}$ & & & & & $x$ & $x$ & & & & \\
\hline
\end{tabular}

Fig. 3 Standard Protocol Items: Recommendations for Interventional Trials (SPIRIT) figure 
therapy) [24]. Incapacitating or life-threatening complications will be classified as serious and will be reported to a clinical research associate. Adherence to treatment will be assessed by providing all patients with a prestructured printed table with all dosages illustrated and then asking them to tick each time a pill was consumed and bring back the table along with any tablet not consumed, which will be counted. In cases of discrepancies between the structured printed table and residual medication, the latter will be taken into account to evaluate the patient's adherence. Good compliance will be defined as the use of at least $80 \%$ of the total medication prescribed.

\section{Data management}

The electronic case report form development and data management for this study will be performed using the Internet-based Clinical Research and Trial (iCReaT) management system (http://icreat.nih.go.kr), which is a data management system established by the Centers for Disease Control and Prevention, Ministry of Health and Welfare, Republic of Korea (iCReaT study number C160025).

\section{Monitoring}

Because the known risks are minimal in this trial, no official data monitoring committee will be established in each study institution. Instead, to maintain the quality of this clinical trial, the clinical research associate will conduct regular monitoring by reviewing the informed consent form, case report form, investigator study file, compliance, serious adverse event, and data records. Monitoring will begin after the first participant completes the whole process of this study. Every institution where the trial is being conducted will be monitored using standard operating procedures while the trial is in process.

\section{Sample size}

Several studies have demonstrated that the eradication rate of standard triple therapy is approximately $75 \%$ in Korea [25]. The eradication rate of sequential or concomitant therapy has been reported to be more than $80 \%[23,26]$. To obtain the optimal eradiation rate, an efficacy greater than $85 \%$ is needed for each treatment [27]. Therefore, we hypothesized that the eradication rate would be superior in the sequential or concomitant therapy groups, with a $10 \%$ difference compared with the rate for standard triple therapy ( $85 \%$ vs. $75 \%)$. To demonstrate this $10 \%$ difference in the eradication rate using a statistical power of $80 \%$ and with the assumption of a one-sided error rate of 0.025 , the protocol requires at least 909 randomly assigned participants. The sample size in this trial was estimated according to the following formula:

$$
n=\frac{\left\{\mathrm{z}_{\alpha} \sqrt{ } 2 P(1-P)+\mathrm{z}_{\beta} \sqrt{ } P_{1}\left(1-P_{1}\right)+P_{2}\left(1-P_{2}\right)\right\}^{2}}{\left(P_{1}-P_{2}\right)^{2}}
$$

To allow for possible dropouts, defined as patients who fail to present or to follow the medication instructions, 1137 subjects will be required.

\section{Statistical analysis}

All efficacy analyses will be calculated for the intentionto-treat (ITT) and per-protocol (PP) populations. The ITT analysis will include all randomized patients who take at least one dose of the study medication. The patients whose infection status is unknown following treatment will be considered as treatment failures for the purposes of ITT analysis. The PP analysis will exclude patients with unknown $H$. pylori status following therapy and those with major protocol violations. The chi-square test, analysis of variance (ANOVA), or Fisher's exact test, as appropriate, will be performed to compare demographic and baseline characteristics between treatment groups. The number and percentage of compliant patients will be tabulated according to the treatment regimen. Differences in compliance rates among treatment regimens will be assessed with 95\% CIs. The chisquare test, ANOVA, or Fisher's exact test, as appropriate, will be performed to compare adverse events and laboratory parameters between groups. The primary endpoint of the study is the $H$. pylori eradication rate after first-line treatment. The secondary endpoints are the frequency of adverse events, treatment compliance, and gastrointestinal symptomatic response after eradication.

\section{Ethics and dissemination}

The study is being conducted in accordance with Declaration of Helsinki standards and Food and Drug Administration regulations regarding good clinical practice. All participants will have been informed of the possible risks and benefits of participating in this clinical trial. After being provided sufficient time to ask questions, participants will be provided an informed consent form that was approved by the local institutional review board. A signed copy of the consent form will be maintained with the study records. Trial participants will be provided a unique participant identification number to maintain confidentiality, and no protected health information will be disseminated. Trial results will be disseminated through scientific conference presentations and by publication in scientific journals.

\section{Discussion}

The Korean College of Helicobacter and Upper Gastrointestinal Research proposed revised guidelines 
in 2013 [10, 28]. These revised guidelines recommend standard triple therapy including a conventional PPI, clarithromycin, and amoxicillin as the primary eradication regimen with a high level of evidence and strong recommendation grade. Furthermore, only this regimen is reimbursed by the Korean government and health insurance system.

However, a recent study using a nationwide Korean database demonstrated that the eradication rate of clarithromycin-based standard triple therapy is 73\% [9]. Furthermore, a comprehensive meta-analysis of Korean reports indicated an overall eradication rate of $74.6 \%$ by ITT analysis and $82.0 \%$ by PP analysis [29]. More importantly, the eradication rate has decreased significantly over the past 10 years [30]. However, this decreasing trend was reported to be different in subgroup analysis, depending on the geographic area. This appeared to be caused by the amount of macrolide antibiotic used [30]. In this respect, clinical guidelines for H. pylori eradication should be modified on the basis of results of a nationwide trial, given the variation in eradication rates after standard triple therapy in each region. Most randomized studies involving the efficacy of first-line treatment were conducted at a single center, and very few multicenter studies have been published to date [29]. A recent nationwide randomized trial revealed that sequential therapy provides higher eradication rates than does standard triple therapy as a first-line therapy in Korea, with ITT eradication rates of $70.8 \%$ for standard triple therapy and $82.4 \%$ for sequential therapy [26]. However, as the authors mentioned, there was a lack of uniformity in the methodologies used among different institutions.

The efficacy of sequential or concomitant therapy for first-line treatment has been reported to be superior to that of clarithromycin-based standard triple therapy by many Korean researchers, which is in agreement with data from other countries. Nevertheless, a pooled analysis showed that sequential treatment led to suboptimal eradication rates in a Korean population, perhaps due to high rates of resistance to clarithromycin and metronidazole [19]. Hence, regional variations in antibiotic resistance need to be understood so that the global optimal protocol for different Korean regions can be determined.

This study is designed to assess the two regimens of greatest interest at present. Furthermore, we will enroll patients from across the different regions of Korea, and a sufficient sample size will be obtained to answer the study question in a statistically rigorous manner. This study will produce vital evidence that will lead to revisions to guidelines concerning first-line treatment regimens for $H$. pylori infection, better eradication rates, and an improved patient quality of life.

\section{Trial status}

Recruitment is ongoing. The first patient was randomly assigned to a treatment group in November 2016. Patient enrollment reached $75 \%$ of target number.

\section{Additional file}

Additional file 1: SPIRIT 2013 checklist: recommended items to address in a clinical trial protocol and related documents. (TIF $3398 \mathrm{~kb}$ )

\begin{abstract}
Abbreviations
ANOVA: Analysis of variance; iCReaT: Internet-based Clinical Research and Trial management system; ITT: Intention to treat; PP: Per protocol; PPI: Proton pump inhibitor; SPIRIT: Standard Protocol Items: Recommendations for Interventional Trials
\end{abstract}

\section{Acknowledgements}

Not applicable.

\section{Funding}

This research was supported by a grant from the Korea Health Technology R\&D Project through the Korea Health Industry Development Institute (KHIDI), funded by the Ministry of Health \& Welfare, Republic of Korea (grant number HI15C1234).

\section{Availability of data and materials \\ Not applicable.}

\section{Authors' contributions}

$\mathrm{HL}$ was responsible for drafting the manuscript, interpreting data, and providing final approval of the manuscript. BJK was responsible for data interpretation and providing final approval of the manuscript. SGK was responsible for the conception and design of the study and for critically revising the manuscript. JGK was responsible for study conception and design, critically revising the protocol, and providing final approval of the manuscript. JJK was the principal investigator responsible for the conception and design of the study, critically revising the protocol, and providing final approval of the manuscript. JIK, IJC, and YCL were responsible for critically revising the protocol and providing final approval of the manuscript. All authors read and approved the final manuscript.

\section{Ethics approval and consent to participate}

We received approval to conduct this study from the institutional review boards of 15 Korean nationwide hospitals: Samsung Medical Center (2016-02-131), Chung-ang University Hospital (C2016106), Yonsei University Severance Hospital (4-2016-0472), National Cancer Center (2016-0185), Bundang Seoul National University Hospital (B-1603/337-008), Chungbuk National University Hospital (2016-02-012), Chungnam National University Hospital (2016-02-020), Chonnam National University Hospital (2016-259), Chonbuk National University Hospital (2016-02-014), Chilgok Kyungpook National University Hospital (2016-03-003), Keimyung University Dongsan Hospital (2016-02-023), Pusan National University Hospital (H-1608-008-044), Kosin University Gospel Hospital (2016-07-034), Jeju National University Hospital (2016-07-007), and Hallym University Chuncheon Sacred Heart Hospital (2016-16). All participants will be provided with written and oral information on the trial so that they can make an informed decision about their participation in the trial. Written informed consent will be obtained from all participants prior to entry into the study.

\section{Consent for publication}

Not applicable.

\section{Competing interests}

The authors declare that they have no competing interests.

\section{Publisher's Note}

Springer Nature remains neutral with regard to jurisdictional claims in published maps and institutional affiliations. 


\section{Author details}

'Department of Medicine, Samsung Medical Center, Sungkyunkwan University School of Medicine, 81 Irwon-ro, Gangnam-gu, Seoul 06351, South Korea. ${ }^{2}$ Department of Internal Medicine, Chung-Ang University College of Medicine, 102 Heukseok-ro, Dongjak-gu, Seoul 06973, South Korea. ${ }^{3}$ Department of Internal Medicine and Liver Research Institute, Seoul National University College of Medicine, Seoul, South Korea. ${ }^{4}$ Department of Internal Medicine, The Catholic University of Korea College of Medicine, Seoul, South Korea. ${ }^{5}$ Center for Gastric Cancer, National Cancer Center Hospital, National Cancer Center, Goyang, Gyeonggi, South Korea. ${ }^{6}$ Department of Internal Medicine, Institute of Gastroenterology, Yonsei University College of Medicine, Seoul, South Korea.

Received: 29 May 2017 Accepted: 25 October 2017

Published online: 17 November 2017

\section{References}

1. Suerbaum S, Michetti P. Helicobacter pylori infection. N Engl J Med. 2002 347(15):1175-86

2. Fock KM, Katelaris P, Sugano K, Ang TL, Hunt R, Talley NJ, Lam SK, Xiao SD, Tan HJ, Wu CY, et al. Second Asia-Pacific Consensus Guidelines for Helicobacter pylori infection. J Gastroenterol Hepatol. 2009:24(10):1587-600.

3. Malfertheiner P, Megraud F, O'Morain CA, Atherton J, Axon AT, Bazzoli F, Gensini GF, Gisbert JP, Graham DY, Rokkas T, et al. Management of Helicobacter pylori infection-the Maastricht IV/ Florence Consensus Report. Gut. 2012;61(5):646-64

4. Asaka M, Kato M, Takahashi S, Fukuda Y, Sugiyama T, Ota H, Uemura N, Murakami K, Satoh K, Sugano K, et al. Guidelines for the management of Helicobacter pylori infection in Japan: 2009 revised edition. Helicobacter. 2010; 15(1):1-20. A published erratum appears in Helicobacter. 2012;17(3):241.

5. Gatta L, Vakil N, Vaira D, Scarpignato C. Global eradication rates for Helicobacter pylori infection: systematic review and meta-analysis of sequential therapy. BMJ. 2013;347:f4587.

6. Kim JM, Kim JS, Jung HC, Kim N, Kim YJ, Song IS. Distribution of antibiotic MICs for Helicobacter pylori strains over a 16-year period in patients from Seoul, South Korea. Antimicrob Agents Chemother. 2004;48(12):4843-7.

7. Malfertheiner P, Megraud F, O'Morain CA, Gisbert JP, Kuipers EJ, Axon AT, Bazzoli F, Gasbarrini A, Atherton J, Graham DY, et al. Management of Helicobacter pylori infection-the Maastricht V/Florence Consensus Report. Gut. 2017:66(1):6-30

8. Lee JW, Kim N, Kim JM, Nam RH, Chang H, Kim JY, Shin CM, Park YS, Lee $\mathrm{DH}$, Jung $\mathrm{HC}$. Prevalence of primary and secondary antimicrobial resistance of Helicobacter pylori in Korea from 2003 through 2012. Helicobacter. 2013;18(3):206-14.

9. Kim BJ, Kim HS, Song HJ, Chung IK, Kim GH, Kim BW, Shim KN, Jeon SW,

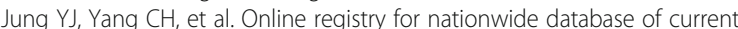
trend of Helicobacter pylori eradication in Korea: interim analysis. J Korean Med Sci. 2016:31(8):1246-53.

10. Kim SG, Jung HK, Lee HL, Jang JY, Lee H, Kim CG, Shin WG, Shin ES, Lee YC, Korean College of Helicobacter and Upper Gastrointestinal Research. Guidelines for the diagnosis and treatment of Helicobacter pylori infection in Korea, 2013 revised edition. J Gastroenterol Hepatol. 2014;29(7):1371-86.

11. Chung JW, Ha M, Yun SC, Kim JH, Lee JJ, Kim YJ, Kim KO, Kwon KA, Park DK, Lee DH. Meta-analysis: sequential therapy is superior to conventional therapy for Helicobacter pylori infection in Korea. Korean J Gastroenterol. 2013;62(5):267-71.

12. Jo HJ, Lee DH, Kang SJ, Kim MN, Kim SH, Park JM, Choi MS, Jung HC, Song IS, Kim N, et al. Comparison of the efficacy of bismuth containing PPI-based quadruple therapy with PPI-based triple therapy only as first-line treatment for Helicobacter pylori infection. Korean J Gastrointest Endosc. 2008;37(4):259-64.

13. Lim JH, Lee DH, Choi C, Lee ST, Kim N, Jeong SH, Kim JW, Hwang JH, Park YS, Lee SH, et al. Clinical outcomes of two-week sequential and concomitant therapies for Helicobacter pylori eradication: a randomized pilot study. Helicobacter. 2013;18(3):180-6.

14. Oh HS, Lee DH, Seo JY, Cho YR, Kim N, Jeoung SH, Kim JW, Hwang JH, Park YS, Lee SH, et al. Ten-day sequential therapy is more effective than proton pump inhibitor-based therapy in Korea: a prospective, randomized study. J Gastroenterol Hepatol. 2012;27(3):504-9.

15. Gatta L, Vakil N, Leandro G, Di Mario F, Vaira D. Sequential therapy or triple therapy for Helicobacter pylori infection: systematic review and meta-analysis of randomized controlled trials in adults and children. Am J Gastroenterol. 2009:104(12):3069-79. quiz 1080.

16. Nyssen OP, McNicholl AG, Megraud F, Savarino V, Oderda G, Fallone CA, Fischbach L, Bazzoli F, Gisbert JP. Sequential versus standard triple first-line therapy for Helicobacter pylori eradication. Cochrane Database Syst Rev. 2016;6:CD009034

17. Kim JS, Kim BW, Ham JH, Park HW, Kim YK, Lee MY, Ji JS, Lee BI, Choi H. Sequential therapy for Helicobacter pylori infection in Korea: systematic review and meta-analysis. Gut Liver. 2013:7(5):546-51.

18. Gisbert JP, Calvet X. Review article: non-bismuth quadruple (concomitant) therapy for eradication of Helicobater pylori. Aliment Pharmacol Ther. 2011;34(6):604-17.

19. Kim JS, Park SM, Kim BW. Sequential or concomitant therapy for eradication of Helicobacter pylori infection: a systematic review and meta-analysis. J Gastroenterol Hepatol. 2015:30(9):1338-45.

20. Graham DY, Lee YC, Wu MS. Rational Helicobacter pylori therapy: evidence-based medicine rather than medicine-based evidence. Clin Gastroenterol Hepatol. 2014;12(2):177-86. e3.

21. Heo J, Jeon SW, Jung JT, Kwon JG, Kim EY, Lee DW, Seo HE, Ha CY, Kim HJ, Kim ES, et al. A randomised clinical trial of 10-day concomitant therapy and standard triple therapy for Helicobacter pylori eradication. Dig Liver Dis. 2014:46(11):980-4.

22. Kim SY, Lee SW, Hyun JJ, Jung SW, Koo JS, Yim HJ, Park JJ, Chun HJ, Choi $\mathrm{JH}$. Comparative study of Helicobacter pylori eradication rates with 5-day quadruple "concomitant" therapy and 7-day standard triple therapy. J Clin Gastroenterol. 2013:47(1):21-4.

23. Lee HJ, Kim JI, Lee JS, Jun EJ, Oh JH, Cheung DY, Chung WC, Kim BW, Kim SS Concomitant therapy achieved the best eradication rate for Helicobacter pylori among various treatment strategies. World J Gastroenterol. 2015;21(1):351-9.

24. Hsu PI, Lai KH, Lin CK, Chen WC, Yu HC, Cheng JS, Tsay FW, Wu CJ, Lo CC, Tseng $\mathrm{HH}$, et al. A prospective randomized trial of esomeprazole- versus pantoprazole-based triple therapy for Helicobacter pylori eradication. Am J Gastroenterol. 2005:100(11):2387-92.

25. Lee SW, Kim HJ, Kim JG. Treatment of Helicobacter pylori infection in Korea: a systematic review and meta-analysis. J Korean Med Sci. 2015;30(8):1001-9.

26. Kim JS, Kim BW, Hong SJ, Kim Jl, Shim KN, Kim JH, Baik GH, Kim SW, Song $\mathrm{HJ}$, Kim JH. Sequential therapy versus triple therapy for the first line treatment of Helicobacter pylori in Korea: a nationwide randomized trial. Gut Liver. 2016;10(4):556-61.

27. Graham DY, Lu H, Yamaoka Y. A report card to grade Helicobacter pylori therapy. Helicobacter. 2007;12(4):275-8.

28. Kim SG, Jung HK, Lee HL, Jang JY, Lee H, Kim CG, Shin WG, Shin ES, Lee YC, Korean College of Helicobacter and Upper Gastrointestinal Research. Guidelines for the diagnosis and treatment of Helicobacter pylori infection in Korea, 2013 revised edition [in Korean]. Korean J Gastroenterol. 2013;62(1):3-26.

29. Gong EJ, Yun SC, Jung HY, Lim H, Choi KS, Ahn JY, Lee JH, Kim DH, Choi KD, Song HJ, et al. Meta-analysis of first-line triple therapy for Helicobacter pylori eradication in Korea: is it time to change? J Korean Med Sci. 2014;29(5):704-13.

30. Shin WG, Lee SW, Baik GH, Huh KC, Lee SI, Chung JW, Jung WT, Park MI, Jung HK, Kim HU, et al. Eradication rates of Helicobacter pylori in Korea over the past 10 years and correlation of the amount of antibiotics use: nationwide survey. Helicobacter. 2016;21(4):266-78.

\section{Submit your next manuscript to BioMed Central and we will help you at every step:}

- We accept pre-submission inquiries

- Our selector tool helps you to find the most relevant journal

- We provide round the clock customer support

- Convenient online submission

- Thorough peer review

- Inclusion in PubMed and all major indexing services

- Maximum visibility for your research

Submit your manuscript at www.biomedcentral.com/submit 